\title{
CAUSAL PARADOXES: A CONFLICT BETWEEN RELATIVITY AND THE ARROW OF TIME
}

\author{
Hrvoje Nikolić \\ Theoretical Physics Division \\ Rudjer Bošković Institute \\ P.O.B. 180, HR-10002 Zagreb, Croatia \\ E-mail: hrvoje@thphys.irb.hr
}

\begin{abstract}
It is often argued that superluminal velocities and nontrivial spacetime topologies, allowed by the theory of relativity, may lead to causal paradoxes. By emphasizing that the notion of causality assumes the existence of a time arrow (TA) that points from the past to the future, the apparent paradoxes appear to be an artefact of the wrong tacit assumption that the relativistic coordinate TA coincides with the physical TA. The latter should be identified with the thermodynamic TA, which, by being absolute and irrotational, does not lead to paradoxes.
\end{abstract}

Key words: causal paradox, relativity, thermodynamic time arrow.

\section{INTRODUCTION - A BRIEF OVERVIEW OF CAUSAL PARADOXES}

The principle of causality says that the past influences the future, while the future does not influence the past. Clearly, this principle assumes the existence of a direction of time, that is, a time arrow (TA) that points from the past to the future. However, according to the theory of relativity, time is not absolute. In particular, the time ordering of points in spacetime depends on the choice of the time coordinate. It is often argued that this relativity of time may lead to causal paradoxes, provided that superluminal propagation of information is possible [1, 2, 3, 4, 5, or that the geometry and topology of spacetime allows closed timelike curves 6, 7, 8, 9]. The superluminal propagation of information may be realized through the propagation of particles with negative squared mass (tachyons) [10, 2, 4, or through a modification of the dispersion relation (induced by interactions) of massless particles [1, 12, 13, 14, 15, 16, 17, 18, A spacetime geometry and topology that allows closed timelike curves may be realized with wormholes that require negative energy [19, 8, 20, and with other mechanisms [6, 7, 21]. There is a lot of work trying to prove the so called chronology protection conjecture [22, 23, 24, 25, 9, 26], which essentially says that that the laws of physics conspire so that the physical conditions needed for a causal paradox cannot realize in nature. However, a convincing proof of this conjecture is still far from being accomplished [27, 28, 29, 9, 30, 31].

The basic idea for a construction of a causal paradox is allways the same. One has to construct a causal loop, that is, a globally oriented closed curve in spacetime, such that the orientation corresponds to the direction from the causes to the consequences. The principle of causality says that the causes influence the consequences, while the consequences do not influence the causes. However, since the curve is closed, it is necessary that a cause eventually becomes a consequence of its own consequence, which may lead to a causal paradox. From the relativistic 
point of view, the paradox can be viewed in the following way. Locally, each point of the causal loop is oriented from the past to the future, as seen by the corresponding local observer. (When the curve is timelike, the "corresponding" observer may be identified with an observer that moves along this curve. When the curve is locally lightlike or spacelike, the "corresponding" observer may be identified with an observer for which this part of the curve is oriented from the past to the future.) However, in a fixed global system of coordinates, some parts of the causal loop may be oriented from the future to the past, while others may be oriented from the past to the future. This means that, along some parts of the curve, the future may influence the past, which contradicts the principle of causality. The aim of this paper is to present a general solution of the causal paradoxes that may appear within the scheme above.

Before starting with a detailed analysis, let us briefly present the conceptual picture for which we present arguments in the subsequent sections of the paper. At the fundamental level, time should be treated on an equal footing with space. In this sense, there can be no time-travel paradoxes, just as there are no space-travel paradoxes. Time does not lapse, just as space does not lapse. Not every initial condition needs to lead to a consistent solution (of an equation of motion), just as not every boundary condition needs to lead to a consistent solution. This is not inconsistent as long as there exist consistent global solutions that are not necessarily found by fixing an initial or a boundary condition. The notion of causality does not make sense at the fundamental level. Without causality, there can be no causal paradoxes. However, in order to explain why many people think that there are causal paradoxes, we need to explain why they think that there is causality, or why they think that time lapses, or why they think that every initial condition should lead to a consistent solution. We find that the origin of all such time-space asymmetric thinkings can be traced back to the existence of the thermodynamic TA, which, in turn, is not a fundamental phenomenon. In other words, since people live in a universe with a thermodynamic TA, it seems to them that there are possibilities for causal paradoxes, despite the fact that in reality there are no possibilities for causal paradoxes at all.

\section{THE ORIGIN OF THE ARROW OF TIME}

The key point of the solution is the fact that the fundamental laws of physics (at least those that we know) do not contain any preferred direction of time. There is no difference between the future and the past at the fundamental level. If the Cauchy problem corresponding to the fundamental equations of motion is well posed, then the initial data on a Cauchy surface determine the physical quantities in the both directions from the Cauchy surface. Therefore, from the fundamental point of view, it is meaningless to say that the past influences the future, but that the future does not influence the past. In this sense, the principle of causality formulated as above is not a fundamental physical principle.

The principle of causality is only an effective phenomenological physical law, valid only on the macroscopic level. The TA pointing from the past to the future exists only on the macroscopic level. There are various phenomenological manifestations of the TA, such as the causal TA, the psychological TA and the electrodynamic TA. However, they all can be reduced to the thermodynamic TA, that is, to the phenomenological rule that entropy (or some other measure of disorder) increases with time 32, 33, 34, 35]. (For popular explanations, see also Refs. 36, 37.) Therefore, the physical TA is the thermodynamic TA.

In particular, the origin of the causal TA can be qualitatively understood as follows. When the Cauchy problem is well posed, then the complete knowledge of the present state determines uniquelly and self-consistently both the future and the past. However, in practice, we try to draw conclusions about the future and the past without knowing details of the present state. Thus, 
our conclusions are to a great extent based on statistical arguments. Since disorder increases with time, statistical arguments can determine the past much better than the future. Therefore, it is typically easier to see a relation between the presence and the past than to see a relation between the presence and the future. This is why, in practice, we consider the past as a cause of the presence, but we do not consider the future as a cause of the presence.

The causal TA is also closely related to the psychological TA, that is, to our psychological experience that time lapses from the past to the future. This experience is a consequence of the rule that brains remember the past, but not the future. As recalling allways reduces to an observation of a present state (of the medium that stores the data) and subsequent conclusion about events that do not refer to the present time, it is clear that the psychological TA reduces to the causal TA. (For a recent detailed discussion of the psychological TA see Ref. 38.)

The fact that we live in a universe in which entropy increases with time means that, in the past, the universe was in a state with a very low entropy. Such a low entropy state is very improbable and we do not know why the nature have chosen such improbable initial conditions. (For a recent attempt to explain this, see Refs. [39, 40. For a general discussion, see, e.g., Ref. [41.) However, the point is that the existence of the thermodynamic TA is merely a macroscopic property of the specific solution in which we live. (In fact, even in an equilibrium where a TA does not exist, the thermodynamic state still defines the physical time in a fully general-relativistic background-independent manner [42, 43.)

\section{RELATIVISTIC DEFINITION OF THE THERMODYNAMIC TA}

In order to give a more quantitative discussion, let us introduce the entropy density $s(x)$ 44, 45], defined everywhere in the universe. It transforms as a scalar under general coordinate transformations. (From this scalar, one can also construct the entropy vector $s^{\mu}=s d x^{\mu} / d \tau$, where $d x^{\mu} / d \tau$ is the macroscopic local 4-velocity of a fluid [44. Alternatively, if one starts from the entropy vector as a more fundamenatl quantity, one can define the entropy scalar as $s=\left|s^{\mu} s_{\mu}\right|^{1 / 2}$.) We can define the local TA in an absolute and relativistically covariant way, as a direction of the gradient of $s$

$$
t_{\mu}=\partial_{\mu} s
$$

In fact, the vector $t_{\mu}$ does not need to be timelike, but we live in a universe in which it is usually timelike, so we retain the name "time arrow" (TA). Along a given curve in spacetime, one can decompose $t_{\mu}$ as

$$
t_{\mu}=t_{\mu}^{\|}+t_{\mu}^{\perp}
$$

where $t_{\mu}^{\|}$is the component of $t_{\mu}$ tangential to the curve, while $t_{\mu}^{\perp}$ is the component normal to the curve. The vector $t_{\mu}^{\|}$defines the local orientation of the curve. The physical TA on the curve is given by $t_{\mu}^{\|}$. If the curve is a timelike trajectory of a local observer, then the TA locally seen by this observer is given by $t_{\mu}^{\|}$, which is a timelike vector. From (2) and (11) we see that the integral of $t_{\mu}^{\|}$over any closed curve is

$$
\oint d x^{\mu} t_{\mu}^{\|}=\oint d x^{\mu} t_{\mu}=0
$$

This implies that a closed curve cannot possess a physical global orientation. Consequently, a causal loop cannot exist, which eliminates a possibility for a causal paradox. It is a simple consequence of the fact that the physical TA is a gradient, which implies that it is irrotational. 


\section{RELATION BETWEEN CAUSAL PARADOXES AND THE THERMODYNAMIC TA}

Now it is easy to understand the source of apparent causal paradoxes. Consider, for example, a closed timelike curve $x^{\mu}(\tau)$, where $\tau$ is an affine parameter on the curve equal to the corresponding relativistic proper length. The orientation of the curve can be chosen to be directed from a smaller to a larger value of $\tau$. The proper length can be interpreted as the proper time of the observer whose trajectory coincides with the curve. Assuming that such an orientation correponds to the physical direction of the proper time, one finds a causal paradox. However, such an orientation is only a coordinate orientation. The true physical orientation is defined

locally by $t_{\mu}^{\|}$. It is impossible that the coordinate orientation above everywhere coincides with the physical orientation. Instead, at some parts of the closed curve, the coordinate TA has the direction opposite to the physical TA.

The explanation above requires some additional clarifications. One may object that there are realistic systems in which the local entropy density decreases with the physical time, which is consistent with the thermodynamical laws which say that only the total entropy of a large isolated system must increase with the physical time. We have two responses to such objections. First, in such systems, the physical time is defined globally, as a coordinate with respect to which the total entropy increases. An observer which only measures the local entropy will naturally assign a different orientation of physical time. (After all, we cannot be sure that in some distant parts of the universe invisible by us the entropy of isolated systems does not decrease with the usual globally defined coordinate time.) Second, for observers that are able to measure entropy in large regions of spacetime, it is natural to divide the spacetime into appropriately large regions and to assign the entropy to each region as the average entropy of this region. This division of spacetime induces also the division of any curve in spacetime. In general, the neighboring regions of a curve have different entropies, which defines the local orientation of a curve on each boundary between two regions on the curve. Again, it is easy to see that such a procedure cannot assign a global orientation to a closed curve, because, if not all regions have the same entropy, there will be at least two boundaries that have relative orientations that are inconsistent with the global orientation. This again solves the causal paradoxes.

Some forms of the causal paradox rest on the fact that, for some spacetime topologies, the Cauchy problem is not well posed [46. The simplest example is a cylindrical spacetime in which the time coordinates $t=0$ and $t=2 \pi$ are identified. As a solution $\phi(t, \mathbf{x})$ of the equations of motion must be periodic such that $\phi(t, \mathbf{x})=\phi(t+2 \pi, \mathbf{x})$, it is clear that not every initial condition leads to a solution. If one assumes that, in principle, any initial condition can be realized in nature, then one may obtain a paradox consisting in finding a solution for which $\phi(t, \mathbf{x}) \neq \phi(t+2 \pi, \mathbf{x})$. At first sight, such forms of the paradoxes have nothing to do with the thermodynamic TA. However, this is not completely true; there is an indirect relation with the thermodynamic TA. Mathematically, the source of the paradox lies in the wrong assumption that any initial condition should lead to a solution. Indeed, if one abandones this wrong assumption, then there is no paradox at all. But the fact that some people think of it as a paradox indicates that there exists some deeper reason for retaining this assumption. To identify this reason, consider a cylindrical spacetime in which it is the space coordinate that is compactified. Now it is the boundary condition (not the initial condition) that cannot be arbitrary. Physicists find nothing paradoxical with having a constraint on the boundary condition. So why they find a paradox when it is the initial condition that is constrained, so that it cannot be arbitrary? Obviously, contrary to the fundamental principle of relativity, they do not treat time on the equal footing with space. But the only physical source of different treatments of space and time 
is the existence of the physical TA, which, of course, is the thermodynamic TA.

For a complete discussion of causal paradoxes, it is unavoidable to mention the relation with the concept of "free will" [4, 9, 46]. Some physicists would like to retain the concept of "free will" compatible with physical laws. They often think of "free will" as something that determines the initial conditions, so they view a constraint on an initial condition as a constraint on "free will", which they often find unsatisfying. However, the usual notion of "free will" that influences the future but not the past is clearly related to the psychological TA, which, in turn, is a consequence of the thermodynamic TA. A notion of a "fundamental free will" compatible with the fundamental physical laws should treat time on the equal footing with space, so there is no any a priori reason why "free will" should act in terms of initial conditions.

At the fundamental relativistic level, time should be treated on the equal footing with space. All physical quantities are described by certain functions $\phi(x)$, where $x$ are points on spacetime. The functions $\phi(x)$ are required to be single valued. (In fact, if they are not single valued, then they are not functions at all.) Clearly, the requirement of single valuedness automatically excludes paradoxes. This requirement is sometimes postulated [47] or derived from other physical laws 48, 49] as the principle of self-consistency, which serves as a general mechanism that prevents causal paradoxes. In our view, the principle of self-consistency is merely a tautology that does not need a separate postulate or an additional justification (see also Ref. [50]). It needs a separate postulate or a justification if one starts from the assumption that the universe is fundamentally determined by its initial condition. However, such an assumption is not relativistic in spirit, but is related to our subjective experience of time that reflects the existence of the thermodynamic TA. Therefore, we abandon this assumption.

\section{CONCLUSION}

Our results can be summarized as follows. The apparent causal paradoxes are an artefact of the wrong tacit assumption that the relativity of time implies also the relativity of the time arrow. Instead, the TA that makes the distinction between the past and the future is absolute, but defined only on the macroscopic phenomenological level. Since the theory of relativity does not allow the existence of an absolute preferred time direction, this constitutes an apparent conflict between relativity and the arrow of time. However, there is no real conflict, because the TA is merely a property of a specific solution of (presumably relativistic) equations of motion that determine our universe.

Acknowledgements. The author is grateful to K. Kumerički for his critical reading of the manuscript and useful remarks. This work was supported by the Ministry of Science and Technology of the Republic of Croatia under Contract No. 0098002.

\section{References}

[1] R. C. Tolman, The Theory of Relativity of Motion (University of California Press, Berkeley, 1917).

[2] O-M. Bilaniuk and E. C. G. Sudarshan, Phys. Today 22, 43 (1969).

[3] G. A. Benford, D. L. Book and W. A. Newcomb, Phys. Rev. D 2, 263 (1970).

[4] E. Recami, Found. Phys. 17, 239 (1987).

[5] A. D. Dolgov and I. D. Novikov, Phys. Lett. B 442, 82 (1998). 
[6] K. Gödel, Rev. Mod. Phys. 21, 447 (1949).

[7] F. J. Tipler, Phys. Rev. D 9, 2203 (1974).

[8] M. S. Morris, K. S. Thorne and U. Yurtsever, Phys. Rev. Lett. 61, 1446 (1988).

[9] M. Visser, gr-qc/0204022.

[10] O. M. P. Bilaniuk, V. K. Deshpande and E. C. G. Sudarshan, Am. J. Phys. 30, 718 (1962).

[11] C. G. B. Garrett and D. E. McCumber, Phys. Rev. A 1, 305 (1970).

[12] S. Chu and S. Wong, Phys. Rev. Lett. 48, 738 (1982).

[13] I. T. Drummond and S. J. Hathrell, Phys. Rev. D 22, 343 (1980).

[14] K. Scharnhorst, Phys. Lett. B 22, 354 (1990).

[15] T. Y. Chiao, Phys. Rev. A 48, R34 (1993).

[16] E. Bolda, R. Y. Chiao and J. C. Garrison, Phys. Rev. A 48, 3890 (1993).

[17] R. Y. Chiao, A. E. Kozhekin and G. Kurizki, Phys. Rev. Lett. 77, 1254 (1996).

[18] N. Bilić and H. Nikolić, Phys. Rev. D 68, 085008 (2003).

[19] M. S. Morris and K. S. Thorne, Am. J. Phys. 56, 395 (1988).

[20] M. Visser, Phys. Rev. D 39, 3182 (1989).

[21] J. R. Gott III, Phys. Rev. Lett. 66, 1126 (1991).

[22] S. W. Hawking, Phys. Rev. D 46, 603 (1992).

[23] S. Deser, R. Jackiw and G. 't Hooft, Phys. Rev. Lett. 68, 267 (1992).

[24] J. D. E. Grant, Phys. Rev. D 47, 2388 (1993).

[25] S. V. Sushkov, Class. Quant. Grav. 14, 523 (1997).

[26] S. Liberati, S. Sonego and M. Visser, Ann. Phys. 298, 167 (2002).

[27] S. W. Kim and K. P. Thorne, Phys. Rev. D 43, 3929 (1991).

[28] T. Tanaka and W. A. Hiscock, Phys. Rev. D 49, 5240 (1994).

[29] S. V. Krasnikov, Phys. Rev. D 54, 7322 (1996).

[30] M. Visser, S. Kar and N. Dadhich, Phys. Rev. Lett. 90, 201102 (2003).

[31] P. K. F. Kuhfittig, Phys. Rev. 68, 067502 (2003).

[32] R. P. Feynman, R. B. Leighton and M. Sands, The Feynman Lectures on Physics (AddisonWesley, Reading, Mass. 1963).

[33] P. C. W. Davies, The Physics of Time Asymmetry (Surrey University Press, London, 1974).

[34] H. Zeh, The Physical Basis of the Direction of Time (Springer-Verlag, 1992). 
[35] J. L. Lebowitz, cond-mat/9605183.

[36] R. P. Feynman, The Character of Physical Law (MIT Press, Cambridge, 1967).

[37] S. W. Hawking, A Brief History of Time (Bantam, London, 1988).

[38] J. B. Hartle, Am. J. Phys. 73, 101 (2005).

[39] S. M. Carroll and J. Chen, hep-th/0410270.

[40] H. Nikolić, hep-th/0411115.

[41] H. Price, gr-qc/9310022.

[42] C. Rovelli, Class. Quant. Grav. 10, 1549 (1993).

[43] A. Connes and C. Rovelli, Class. Quant. Grav. 11, 2899 (1994).

[44] R. C. Tolman, Relativity, Thermodynamics and Cosmology (Clarendon Press, Oxford, 1934).

[45] N. Bilić and H. Nikolić, Nucl. Phys. B 590, 575 (2000).

[46] J. Friedman, M. S. Morris, I. D. Novikov, F. Echeverria, G. Klinkhammer, K. S. Thorne and U. Yurtsever, Phys. Rev. D 42, 1915 (1990).

[47] I. D. Novikov, Phys. Rev. D 45, 1989 (1992).

[48] A. Carlini, V. P. Frolov, M. B. Mensky, I. D. Novikov and H. H. Soleng, Int. J. Mod. Phys. D 4, 557 (1995).

[49] A. Carlini and I. D. Novikov, Int. J. Mod. Phys. D 5, 445 (1996).

[50] D. Deutsch, Phys. Rev. D 44, 3197 (1991). 